\title{
La persona y el Estado como agentes de responsabilidad para la producción de sociedades saludables: análisis temático desde la perspectiva de profesionales de la salud en Cataluña (España)
}

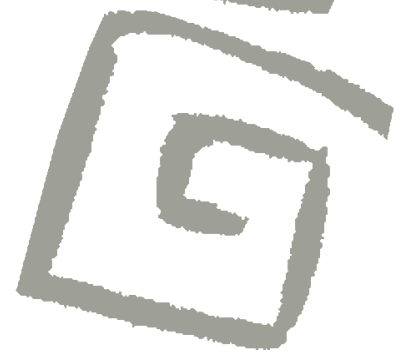

${ }^{\circledR}$ Doctora en Psicología. Profesora asociada, Departamento de Psicología Social, Universidad de Barcelona, España. $\bowtie$ iD
The individual and the State as agents responsible for the production of healthy societies: a thematic analysis from the perspective of health professionals in Catalonia (Spain)

\section{Valeria Santoro Lamelas ${ }^{1}$}

RESUMEN El objetivo del artículo es analizar los sentidos a partir de los cuales se articulan las prácticas de intervención en promoción de la salud y sus consecuencias en la atribución de responsabilidades en salud. La aprobación de la Ley 18/2009 de Salud Pública de Cataluña propició la realización del Projecte demostratiu de l'Agència de Salut Pública de Catalunya el cual incluyó, durante 2009 y 2010, el Pla transversal d'educació i promoció de la salut en infants i joves en La Garrotxa (comarca de Cataluña), marco en el que se realizaron 20 entrevistas a informantes claves. Mediante un análisis temático, encontramos que se identifica al Estado y a la persona como los principales agentes responsables de la producción de sociedades saludables. Asimismo, evidenciamos que, a partir de diferentes articulaciones entre los discursos que refieren a la toma libre y racional de decisiones, y aquellos que refieren al entorno social, político y económico, se configuran diferentes enfoques sobre la responsabilidad, cuyos efectos se relacionan con la potenciación (o no) del Estado como garante de la salud poblacional en contraste con la culpabilización personal.

PALABRAS CLAVES Salud Pública; Promoción de la Salud; Toma de Decisiones; Responsabilidad Social; Conocimientos, Actitudes y Práctica en Salud; España.

\begin{abstract}
This article aims to analyze the meanings upon which health promotion intervention practices are based, and the consequences of these meanings in the identification of responsibilities in health. The passage of Catalonia's Public Health Law 18/2009 facilitated the development of the Demonstrative Project of the Public Health Agency, in the framework of which fieldwork for the Plan for Health Education and Promotion in Children and Adolescents in La Garrotxa (region of Catalonia) was carried out. In this way, 20 interviews with key informants were conducted. Through a thematic analysis, it was found that the State and the individual are identified as the primary agents responsible for the production of healthy societies. It was also evidenced that, in the articulation between the discourses referring to free and rational decisionmaking and those referring to the social, political and economic environment, different approaches towards responsibility are construed, with effects related to the potentiation (or lack thereof) of the State as a guarantor of the population's health in opposition to blaming of the individual.
\end{abstract}

KEY WORDS Public Health; Health Promotion; Decision Making; Social Responsibility; Health Knowledge, Attitudes, Practice; Spain. 


\section{INTRODUCCIÓN}

En Cataluña (Comunidad Autónoma Española), las nociones actuales sobre salud pública están estrechamente ligadas al discurso globalizado de las sociedades opulentas y envejecidas. Es por este motivo que los problemas de salud pública se asocian a las ideas de progreso y globalización, y los principales focos de atención de las acciones gubernamentales en salud se orientan al aumento de la esperanza de vida, el medio ambiente y los hábitos personales ${ }^{(1)}$.

En este marco, la promoción de la salud adquiere especial fuerza como área de la salud pública orientada a mejorar las condiciones de vida y salud de la población y a evitar y/o reducir el impacto de los efectos negativos del "progreso". Para alcanzar sus objetivos, la promoción de la salud plantea la importancia de pensar la salud como un proceso que tiene que ser producido y reproducido en todos los espacios y momentos de la vida de las personas ${ }^{(2)}$, trascendiendo, de esta manera, la acción en salud de los ámbitos puramente sanitarios hasta penetrar en cada espacio de vida y relación de las personas ${ }^{(3)}$.

Esta perspectiva de la salud es una de las condiciones de posibilidad para el desarrollo de las "sociedades saludables", un proyecto de sociedad en el que la salud se hace presente en cada espacio relacional ${ }^{(4,5)}$. Dicho en otras palabras, sociedades que se responsabilizan en desarrollar estrategias de gobierno con la finalidad de mejorar la salud de su población mediante la promoción de estilos de vida saludables y el despliegue de políticas que incidan sobre el entorno, entendido como marco material y social que puede potenciar o perjudicar la salud.

Se propone, pues, el desarrollo de un modelo de sociedad basado en el despliegue de múltiples tecnologías de saber e intervención en salud pública, en el cual nada se escapa a las políticas de salud, ya que todos los ámbitos de existencia y relación son susceptibles de ser tratados en aras de mejorar la salud de la población. En este contexto, las sociedades saludables son la expresión de una forma específica del biopoder ${ }^{(6,7)}$, entendido como un ejercicio del poder que fundamenta sus principios de gobierno en la salud y la vida, y la promoción de la salud como una estrategia biopolítica ${ }^{(8,9)}$ que genera saberes, especialistas e intervenciones sobre modelos de vida, de salud y de relaciones esperables, legítimas y eficaces ${ }^{(10)}$.

Dentro de la línea de desarrollo de sociedades saludables, con la aprobación de la Ley 18/2009 de Salud Pública(11), se desplegó en Cataluña una reforma normativa e institucional de la salud pública que ha promovido un nuevo modelo de salud pública que destaca la relevancia de la multiplicidad de elementos que condicionan la salud, plantea la necesidad de integrar agentes gubernamentales del área de la salud junto con otras áreas gubernamentales (como educación, urbanismo, deportes, etc.) y se propone abrir la participación a instituciones no gubernamentales (ONG, empresas, consorcios, etc.) en el desarrollo de intervenciones.

En Cataluña, la Ley 18/2009 de Salud Pública, da lugar a la creación de la Agència de Salut Pública de Catalunya (ASPCAT). Para su desarrollo e instauración territorial, en el período 2009-2010 se implementaron proyectos piloto con el objetivo de probar la ejecución del nuevo modelo. En especial se desarrollaron proyectos en el área de promoción de la salud, dada su relevancia en la construcción de sociedades saludables, en un territorio en el que la protección de la salud y la vigilancia epidemiológica se encuentran arraigados.

Concretamente, la investigación que presentamos se enmarca en el Projecte Demostratiu de l'Agència de Salut Pública de Catalunya, el cual incluyó el Pla transversal d'educació i promoció de la salut en infants $i$ joves en La Garrotxa, realizado en el período 2009-2010. Para el despliegue del proyecto se tomó como principio básico la participación de diversas entidades y agentes en el área de promoción de la salud siguiendo el modelo de gobernanza presentado por el Departament de Salut. Este modelo destacaba el trabajo articulado desde el nivel central, regional y territorial, y contaba con la 
participación de entidades territoriales como el ayuntamiento, la escuela y el Consorcio de Salud, entre otras. Asimismo, se planteó un trabajo transversal con agentes de diversas disciplinas de salud y de educación. Todas estas relaciones y articulaciones se realizaron destacando la necesidad de que cada organización y área, en la búsqueda de colaboración y de complementariedad de actuaciones, de ideas y de recursos, mantuviese su autonomía organizativa.

Así pues, a partir de este marco, hemos desarrollado la investigación que dio lugar a la tesis doctoral "La salud pública como problema de gobierno: Análisis de los problemas sociales de salud pública bajo el modelo de gobernanza", con la finalidad de conocer cómo la salud pública se constituye como una tecnología de gobierno sobre determinados problemas sociales. Del trabajo desarrollado en la tesis doctoral derivan el artículo "La salud pública en el continuo salud-enfermedad: un análisis desde la mirada

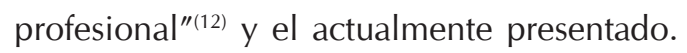
En ambos trabajos se abordan dos componentes diferenciados: cómo se construye la salud pública como área de intervención dentro de las políticas públicas de salud y quiénes son los sujetos de responsabilidad en la construcción de sociedades saludables. Así pues, aunque ambos artículos comparten la metodología y los informantes, difieren en la temática tratada (reflejados en la divergencia de referencias utilizadas, los resultados expuestos, sus conclusiones y discusión). Por su parte, en el artículo "La salud pública en el continuo salud-enfermedad: un análisis desde la mirada profesional" identificamos al continuo salud-enfermedad como elemento clave utilizado por las y los profesionales de salud para constituir y posicionar las diversas políticas e intervenciones del Estado sobre la salud de su población, y debatimos sobre las implicancias de pensar la salud a partir de la multiplicidad de sus causas y del bienestar en el diseño de políticas de salud pública. Por el contrario, en este artículo, nos propusimos analizar cuáles son los sentidos a partir de los cuales se articulan las prácticas de intervención de promoción de la salud y sus efectos en la construcción de sujetos de responsabilidad en salud. Nos adentramos pues, en el proceso de responsabilización de sujetos e instituciones para la construcción de "sociedades saludables" y las consecuencias de adoptar enfoques personales o comunitarios.

\section{METODOLOGÍA}

Desde la adscripción a la perspectiva construccionista, entendemos que no existe realidad ajena (o externa) a la experiencia lingüística del mundo ${ }^{(13)}$, producida a través de las prácticas colectivas, siempre social e históricamente situadas ${ }^{(14,15)}$. Desde nuestro posicionamiento, el lenguaje no tiene solo una función referencial (describir y expresar una experiencia) sino que es de naturaleza performativa y formativa. La naturaleza perfomativa implica que el lenguaje está orientado a la acción ${ }^{(16)}$. La naturaleza formativa refiere a que hablar implica crear realidades. Es decir, los sentidos, las prioridades, los intereses, etc., se producen a partir de un entramado de estructuras y categorías sociales preexistentes que, a su vez, en la misma práctica lingüística, producimos, reproducimos y/o cambiamos $^{(17)}$.

Nuestro posicionamiento epistemológico requirió de la elección de una metodología que permitiera analizar los procesos de producción de sentidos de forma profunda y específica, teniendo en cuenta la complejidad y dinamismo de los procesos sociales. Por este motivo, planteamos el desarrollo de una investigación cualitativa, la cual permite un acceso riguroso, válido y fiable a los procesos de interacción social, la producción de significados y la confrontación y/o negociación de argumentos, poniendo especial atención en las personas involucradas en tales procesos $y$ en los contextos en los que estos son producidos. Así pues, hemos adoptado una metodología cualitativa, en primer lugar, por su orientación hacia un diseño metodológico abierto y flexible, que permite la producción e integración de informaciones emergentes y 
no estereotipadas sobre los sentidos articulados en torno a la promoción de la salud y, en segundo lugar, por las potencialidades que dicha metodología ofrece en la comprensión de tales sentidos a partir de un análisis profundo, complejo y situado ${ }^{(18,19)}$.

En la tarea de acceso y análisis del proceso de construcción de la promoción de la salud, destacamos la importancia que detenta el lenguaje en la totalidad de la investigación; tanto para el acceso a la producción de significados, como para el análisis de los discursos vinculados a su producción.

\section{Informantes y técnica de recogida de información}

Tal como se detalló previamente, efectuamos el trabajo de campo de esta investigación en el marco del desarrollo del Pla transversal d'educació i promoció de la salut en infants $i$ joves en La Garrotxa, uno de los Projecte Demostratiu de l'Agència de Salut Pública de Catalunya, que tenía como finalidad servir como punto de inicio para la producción de nuevas estrategias de promoción de la salud fundamentadas en la coordinación entre los diversos agentes de salud de la comunidad autónoma y del nivel local de acuerdo con la propuesta de acción coordinada en salud de la Ley 18/2009 de Salud Pública de Cataluña.

Para la recopilación de la información analizada en esta investigación, realizamos entrevistas individuales, dado que esta técnica se produce en un marco de conversación pautada que permite acceder a información más detallada sobre sucesos y situaciones de escenarios y momentos diversos. A su vez, su carácter comunicativo, hace posible captar significados complejos, mediados por la construcción que las personas elaboran de los acontecimientos en el acto comunicativo de contar su experiencia ${ }^{(20,21)}$.

Concretamente, realizamos 20 entrevistas a informantes claves, cuya selección se basó en la identificación de uno de los equipos motores del proyecto, el autodenominado "equipo operativo", compuesto por agentes de salud de niveles centrales, regionales y territoriales. Este equipo operativo era el encargado de dirigir el curso del proyecto, analizar propuestas de intervención y reformularlas en aras de garantizar la ejecución del proyecto. Así pues, realizamos entrevistas a todos los miembros activos del equipo operativo y a otros agentes que, aunque no formaran explícitamente parte de este equipo, participaban en él de forma directa y activa.

Las entrevistas se realizaron en catalán o en castellano, según la preferencia de habla de las personas entrevistadas, y los extractos de las entrevistas en catalán fueron traducidos para la exposición de los resultados en el presente artículo. Las entrevistas tuvieron lugar en el entorno de trabajo habitual de las personas (Barcelona, Girona y La Garrotxa), se efectuaron en el marco del proyecto demostrativo, durante los años 2009 y 2010, y una vez finalizado, durante los años 2011 y 2012 , con el propósito de reflexionar y profundizar sobre aspectos anteriormente tratados.

Al comenzar se le comunicó a cada informante los objetivos de la entrevista, el tratamiento de los datos y la garantía del anonimato de las personas participantes. Cada una/o de ellas/os aceptaron participar de la investigación. Asimismo, contamos con el aval institucional de la Agència de Salut Pública de Catalunya y la Universidad Autónoma de Barcelona para el desarrollo del proyecto, para el cual se constituyó un comité científico de seguimiento con el fin de asegurar el adecuado desarrollo de la investigación, garantizando el cumplimiento de los principios éticos y la rigurosidad científica. La duración media de las entrevistas fue de una hora (con duraciones que oscilaban entre 45 minutos y hora y media).

Los temas contemplados en el guión de las entrevistas abordaban los fundamentos de la salud, la conceptualización de la salud pública y sus áreas, la identificación de los principales problemas de salud pública, las responsabilidades en salud, los motivos para promocionar la salud, los principales elementos de intervención en promoción de la salud y la identificación de sujetos de intervención y responsabilidades en salud. 
Los códigos de clasificación de las entrevistas son:

- Área profesional de la persona entrevistada: salud pública general (SP), promoción de la salud $(\mathrm{Pm})$, protección de la salud (Pt), vigilancia epidemiológica (V), CatSalut (aseguradora pública de salud de Cataluña) (CS), educación (E).

- Nivel de descentralización de su trabajo: nivel autonómico (NA), nivel regional (NR) y nivel territorial (NT).

- Formación profesional: biología (B), docente $(\mathrm{D})$, medicina $(\mathrm{M})$, farmacia $(\mathrm{F})$, veterinaria $(\mathrm{V})$ y medioambiente (MA).

\section{Análisis}

Se planteó la realización de un análisis temático ${ }^{(22)}$ con el propósito de comprender los significados producidos por las y los agentes involucrados con los programas de salud pública, mediante sus intercambios lingüísticos y la exposición de sus posturas ante los asuntos relevantes, y elucidar los efectos de la producción de tales significados.

Una vez que se transcribió la totalidad del corpus, a partir de las lecturas repetidas del conjunto de materiales, iniciamos una aproximación preliminar hacia la codificación de temas y subtemas -es decir, la identificación de segmentos de sentido en el texto de relevancia potencial para el análisis ${ }^{(23)}{ }_{-}$, prestando atención principalmente a la dimensión más descriptiva y subordinando en una segunda fase, la dimensión argumentativa. A continuación, emprendimos una primera categorización de la información, bajo la directriz de mantener, por una parte, el contexto proporcionado por el corpus documental global (conjunto de entrevistas) y su vertebración interpretativa con el núcleo de enunciados que daban cuenta de una misma temática y que permitían un análisis específico y pormenorizado en cada enunciado según temática. En este proceso realizamos tres operaciones: a) elaboración de mapas conceptuales de cada tema y subtemas, identificando también sus relaciones con otros temas; b) descripción de cada tema o posible categoría a partir de la recopilación de las afirmaciones realizadas de forma explícita por las/os informantes, limitando la interpretación de connotaciones implícitas a comentarios añadidos en forma de notas externas y agrupaciones temáticas; c) constatación del vínculo de cada tema con los objetivos de la investigación, con el objetivo de aumentar la receptividad de significados no previstos en los objetivos de la investigación, para lo cual identificamos temas de forma eminentemente inductiva y establecimos vínculos de cada tema y subtema con los objetivos de la investigación.

Con los resultados parciales de las tres operaciones mencionadas (mapas conceptuales, descripción de cada tema o posible categoría y confirmación del repertorio de temas y subtemas emergentes) volvimos al texto original e iniciamos una nueva etapa analítica cuyo objetivo ya no era describir qué se decía, sino comprender cómo operaban los enunciados del texto al construir los objetos de los que hablan, sus relaciones y sus efectos. Para ello:

a. Esclarecimos las posiciones de cada profesional entrevistado/a respecto de la definición de salud pública, y los argumentos comunes y divergentes que se crean desde cada una de estas posiciones y al interior de ellas.

b. Buscamos connotaciones que aparecen de forma reiterativa para justificar, describir, argumentar, etc., la promoción de la salud, y abordamos el porqué de estas reiteraciones, los sentidos que adquirían, los vínculos con otros discursos y sus funciones.

c. Identificamos objetos (salud, hábitos, saberes, etc.) y sujetos (personas, el Estado) referidos en los enunciados.

d. Indagamos sobre los efectos de los discursos, y analizamos las consecuencias de las construcciones de los sujetos y objetos en la asignación de responsabilidades en salud y la atribución de libertades.

En el proceso de análisis, la variabilidad de argumentos nos permitió configurar una 
imagen compleja de la promoción de la salud, al mismo tiempo que nos permitió identificar regularidades y vínculos entre ellos. Así pues, los temas y subtemas adquirieron formas de categorías y subcategorías y se interconectaron, dando un sentido más amplio al fenómeno estudiado, al conformar un sistema argumentativo que permite entender cómo la promoción de la salud es constituida como estrategia de intervención para la construcción de sujetos hegemónicamente saludables.

\section{RESULTADOS}

Los resultados que presentamos permiten comprender las lógicas de intervención en promoción de la salud y la atribución de responsabilidades en salud a partir de un entendimiento específico del sujeto y el entorno. Para ello, estructuramos los argumentos que conforman esta categoría en torno a cuatro segmentos de sentido:

1.La configuración del Estado y las personas como agentes de responsabilidad para la construcción de sociedades saludables.

2.El diseño de intervenciones orientadas a incidir sobre la toma de decisiones personales.

3.El entorno como modulador de las prácticas en salud.

4. La convivencia de enfoques contrapuestos sobre la responsabilidad y sus efectos pragmáticos y éticos.

\section{El Estado y la persona: agentes de responsabilidad para la construcción de sociedades saludables}

Las y los profesionales de salud pública identifican al Estado y a las personas como los principales sujetos de acción en la salud colectiva. Por su parte, asumen que las instituciones públicas, como parte extensa del Estado de Bienestar, tienen una responsabilidad ineludible hacia la población en la identificación de necesidades y el diseño e implementación de intervenciones sobre la salud de la población. En este sentido, mientras crean y transmiten saberes (discursos oficiales de salud convertidos en verdades), las instituciones públicas diseñan y ejecutan intervenciones orientadas a cambiar situaciones, condiciones de vida y prácticas con miras a construir una sociedad hegemónicamente saludable.

\begin{abstract}
Yo creo que la responsabilidad de educar [...] es de las instituciones [...] tú les tienes que ofrecer, si lo agarran o no lo agarran, eso ya es otra historia, o si quieren o no quieren es otro problema ¿no? Entonces ofrecerlo, yo creo que sí, que es responsabilidad de esta parte, no es de ellos ¿no? (CS/NR/M)
\end{abstract}

Destacan el rol de liderazgo de las instituciones gubernamentales de salud pública en dirigir y planificar la salud poblacional. Sin embargo, expresan que la producción de salud trasciende las fronteras del Departamento de Salud hacia diversos ámbitos de la vida cotidiana, donde existen múltiples instituciones públicas con finalidades de gobierno. Como consecuencia, asumen que la incumbencia de la salud no es solo responsabilidad de los departamentos específicamente encargados de ella, sino que trasciende y desborda hacia otras áreas institucionales (educación, urbanismo, medio ambiente, servicios sociales, etc).

Departamento de Salud, vivienda, trabajo, educación, medioambiente, urbanismo, etc., ¿no?, todos los elementos que tienen que ver con realizar políticas que van a impactar sobre la salud pública. (SP/ NA/M)

Siempre hemos dicho que quien planifica es el Departamento de Salud. (CS/ $\mathrm{NR} / \mathrm{M}$ )

Aunque al Estado, en todas sus representaciones institucionales, se le atribuye un rol fundamental en la detección de necesidades, 
la planificación estratégica y el diseño de intervenciones en salud, las y los profesionales refieren a la complejidad social y reconocen la existencia de intervenciones de entidades no gubernamentales y su impacto en la salud poblacional. Es decir, el acento en la multiplicidad de elementos que configuran la salud de la población deriva del hecho de defender la necesidad de que cada una de las instituciones inmersas en el entramado social se comprometa a garantizar la salud de la población.

Se agregan pues ONG, instituciones, asociaciones, el tejido social que también está preocupado por la salud. (SP/NA/M)

Por su parte, apoyándose en la noción de libertad individual, las y los profesionales de la salud le conceden al sujeto la autonomía para asumir riesgos a partir de la ponderación que este haga entre los costos y beneficios de su conducta. Depositan en cada habitante la obligación última del cuidado de su propia salud puesto que, argumentan, más allá de los condicionantes existentes, es quien toma la decisión de hacer o no una práctica saludable o no saludable.

Tú tienes la libertad, tú tienes que poder escoger, tú tienes que ser autónomo, tienes que ser responsable. (Pt/NT/MA)

Asumen que el discurso oficial sobre la salud, promovido por los estamentos científicamente legitimados, es el que debería guiar la práctica personal en salud. Pero reconocen que tal discurso oficial se sumaría al entramado de conocimientos y de experiencias que la persona posee y a partir del cual toma decisiones en sus prácticas, convertidas en hábitos, si se interiorizan en su cotidianeidad. Esto plantea la importancia de incrementar la fuerza del discurso oficial en salud, para garantizar conocimientos y prácticas de salud "correctas".

Quiero decir, tarde o temprano el individuo tendrá... se le presentará una opción y el tendrá que decidir ¿vale? [...] lo que se tiene que hacer es, entonces, potenciar estos argumentos para que cuando sea el momento que le toque decidir, que tenga todos los conocimientos. (Pt/NR/F)

En este sentido, las y los profesionales de salud pública relativizan la responsabilidad del Estado al destacar la importancia de evitar que la población asuma un rol pasivo respecto a su salud, y promocionan la importancia de la toma de decisiones particulares en el cuidado propio para evitar que la responsabilidad final caiga en las instituciones del Estado.

Primero has de asumir la responsabilidad sobre tu salud, es tuya ino? y esto también lo dice la, la Ley de Salud Pública de Cataluña, ¿no? O sea la idea es tratar de transmitir la... las decisiones sobre tu salud que tú tomas, ¿no? (SP/NA/M)

Pero, al mismo tiempo, las y los profesionales de salud pública manifiestan que los límites de la libertad y de la autonomía se vuelven difusos ya que, a su juicio, se produce una tensión en constante movimiento para establecer los límites de vigilar o velar por la salud de la población en la búsqueda de no ser excesivamente paternalistas o excesivamente laxos en la función del Estado. Afirman que el Estado muchas veces aplica medidas correctivas a las desigualdades sociales provocadas por sus propias políticas o por injerencias del mercado (acciones dirigidas a los determinantes de la salud, campañas de educación de hábitos saludables frente a una vorágine de discursos sobre el consumo, la estética, la competitividad, la rapidez, etc.) que fomentan prácticas contrarias a las propuestas para garantizar la salud poblacional.

\footnotetext{
O sea que la idea, claro mucha gente dice "ostras esto es mucho control social", pero ¿y dónde está la libertad individual? pero es que la libertad individual sin información, no sé si es mucha libertad, porque a lo mejor estás condicionado por modas, por elementos comerciales, por no sé qué, ¿no? (SP/NA/M)
} 
Sintéticamente, puede afirmarse que el discurso de las y los profesionales de salud pública sobre la responsabilidad en salud parte de la consideración del Estado como una garantía de cuidado de la salud poblacional, ya que es este quien provee de herramientas y condiciones para que cada ciudadana/o tome las decisiones correctas. En este marco, colocan al sujeto racional como la clave de intervención porque puede reproducir (o no) ciertas prácticas vinculadas con la salud. La toma de decisiones recae en las personas, decisiones entendidas como resoluciones adoptadas a partir del discernimiento del sujeto pensante sobre lo que está bien y lo que está mal y sus consecuencias. Mediante la aplicación de esta lógica, las y los profesionales entrevistados apelan a un cierto grado de libertad atribuida a los actos perpetrados por cada persona cada vez que escoge opciones de ser.

\section{Diseño de intervenciones orientadas a incidir sobre la toma de decisiones personales}

Las y los profesionales de salud pública, al comprender las prácticas personales como un modelo centrado en el sujeto libre y racional, capaz de tomar sus decisiones, y al asumir que la construcción de sociedades saludables se basa en la producción de sujetos con hábitos de salud, destacan la importancia del Estado en proveer conocimientos para guiar las prácticas de salud de las personas.

Desde esta óptica expresan que para orientar las prácticas de salud de las personas es necesario diseñar intervenciones con el objetivo de incidir sobre tres componentes de la acción: la producción de saberes sobre qué hacer para ser saludable (conocer cuáles son las prácticas saludables y cuáles no lo son); la producción de saberes sobre cómo hacerlo (dominar las estrategias para alcanzar las prácticas saludables); y, finalmente, la producción de intenciones de hacerlo (desear realizar las prácticas saludables).
Tienes que saber, tienes que saber hacer y tienes que querer hacerlo en un entorno que te ayude a hacerlo. (Pm/NA/M)

En relación con el primer componente, bajo la argumentación de que son las instituciones públicas quienes deben producir verdades en salud y hacerlas visibles frente a otras informaciones irreales o sesgadas que puedan distorsionar la adquisición de hábitos saludables, las y los profesionales de la salud afirman que la producción de verdades y la diseminación de los discursos sobre lo saludable y lo no saludable son goznes esenciales mediante los cuales las y los profesionales de salud pública articulan las intervenciones de promoción de la salud.

Se tiene que utilizar los medios más eficientes para conseguir que todo lo que es una información que en realidad no es información, sino que es desinformación que tienen los jóvenes. Les llegue cual es la información real. (V/NR/M)

Con respecto al segundo componente, las y los profesionales de la salud asumen que ser saludable es un proceso que "se hace" y, en ese "hacer-se" las prácticas cotidianas son fundamentales para garantizar la producción de personas saludables o, lo que es lo mismo, personas que actúan habitualmente siguiendo los criterios de salud propuestos por los organismos competentes. Para ello, expresan que no basta con que las personas sepan cuál es la norma para ser saludables sino que es necesario que puedan asociar a cada una de esas normas ciertas instrucciones que les permitan alcanzar las metas en salud. Por este motivo, convierten el segundo elemento, el cómo, en otra bisagra esencial para la promoción de la salud. Entienden que el aprendizaje de pautas de acción es una herramienta que habilita a las personas y les proporciona aptitudes para hacer(se) saludables. Es decir, para la producción de sociedades saludables, las personas que conforman tal sociedad deben saber cuáles son las metas en salud (una dieta equilibrada, limitar el consumo de sal y 
grasas saturadas, hacer ejercicio físico, etc.) $y$, al mismo tiempo, deben conocer cuáles son las estrategias para alcanzarlas (cómo se debe cocinar, cómo limitar el consumo de alimentos que tienen más sal o grasas saturadas, cómo integrar el ejercicio físico en el curso de la vida, etc.).

La gente tiene que saber que nosotros cuando hablamos de alimentación saludable no hablamos solo de la comida a la plancha... que puede ser muy variado y que puede ser muy diferente... y que un frito a veces tampoco está mal, si está bien frito, con el aceite adecuado, a la temperatura adecuada, y todo eso. (Pm/NA/M)

Dada la enorme tradición en diseminar información sobre qué es ser saludable, las y los profesionales de salud pública asumen que la población tiene un gran conocimiento sobre los criterios de salud propuestos. No obstante, expresan que las personas tienen un menor conocimiento sobre cuáles son las pautas de acción orientadas a alcanzar tales criterios de salud. Por ello, en las entrevistas destacan la necesidad de desarrollar con mayor ímpetu políticas de promoción de la salud que doten a las personas de saberes estratégicos para actuar con la finalidad de alcanzar la meta de salud.

El problema, muchas veces está en el cómo para alguna gente... es decir. ¿Cómo puedo hacer actividad física si no puedo ir al gimnasio tres veces por semana? Por lo tanto, por eso nuestros mensajes van mucho en decir: ¡no!, tienes que acumular treinta minutos $y$ puede ser... pues eso... bajándote una parada antes del bus... subiendo más las escaleras, haciendo [...], eso iría a saber cómo (Pm/NA/M)

Desde el punto de vista de las y los profesionales de salud pública, la vida se vuelve un conjunto de instrucciones sobre usos prohibidos (por ejemplo, consumir drogas o tabaco, mantener sexo esporádico sin protección, etc.) y sobre matices y formas de ser (qué, cómo, cuándo y cuánto beber, comer, movernos, relacionarnos, etc).

\section{Los cuatro ejes básicos para la promoción de la salud de la población... Cómo nos relacionamos, cómo comemos y cómo nos movemos [...] bueno, y qué consu- mimos. (SP/NA/M)}

Sin embargo, aunque las y los profesionales hacen referencia a ciertos comportamientos idealmente prohibidos (como fumar o drogarse), suelen desplazar los mensajes de la prohibición a la moderación. En efecto, en las entrevistas afirman que promocionar hábitos saludables implica una cierta tolerancia de acciones que, desde la salud pública pueden considerarse no saludables. Manifiestan que no se trata de un planteamiento dicotómico (sano/no sano) en el que todas las acciones potencialmente dañinas sean prohibidas, sino de un continuo en el que ciertas prácticas pueden moderarse hasta ser redirigidas hacia prácticas aceptablemente saludables.
Yo insisto mucho y aún el otro día me llevaba un folleto... que era de fuera de aquí, que decía "folleto de cómo tener una vida saludable: no fumes, no tomes el sol..." y yo decía: "hombre, no! Eso es imposible... ino!". (Pm/NA/M)

Además, al comprender que "ser saludable" se materializa en las acciones producidas desde diversos escenarios, relaciones y momentos de la vida de las personas, argumentan que en cada persona coexisten prácticas que pueden ser consideradas saludables y otras que no lo son tanto. Por este motivo, destacan la importancia de maximizar las conductas del polo saludable, mientras que relegan aquellas que no lo son al terreno de la excepcionalidad para obtener, como resultado de la sumatoria de conductas saludables y no saludables, una vida en la que se transite con un mayor número de prácticas saludables. 
...no, un día no pasa nada, no un día no pasa absolutamente nada, pero un día detrás de otro, es cuando comienza a pasar. (Pt/NT/MA)

Con relación al tercer componente, las y los profesionales manifiestan la necesidad de intervenir para producir la voluntad de ser saludables, en la medida en que argumentan que las prácticas de salud trascienden al propio conocimiento. Entienden que el hecho de albergar la intención, que es otra manera de expresar que existe la voluntad de actuar, está mediado por el conocimiento sobre qué prácticas son saludables, cómo alcanzarlas y también por la valoración del costo-beneficio de estas. Vinculan el beneficio obtenido mediante la práctica de salud con una gran variedad de elementos: desde aspectos físicos (como el incremento de capacidad aeróbica), materiales (como el ahorro económico), interpersonales (como el reconocimiento por el grupo) hasta aspectos ideológicos (como la asociación de la droga con la adicción y esta con la pérdida de libertad).

Dejar de fumar, a lo mejor es una liberación. (SP/NA/M)

Asimismo, manifiestan que el costo tiene una traducción a limitaciones en el entorno de carácter material (esfuerzos para el acceso a ciertos espacios y productos), temporal (disponibilidad de tiempo y necesidad de tiempo requerido para una actividad) o interpersonales (en especial, destacan la presión grupal hacia ciertas prácticas que se vinculan al grupo de pertenencia).

Quiero decir, mensajes negativos o positivos, por ejemplo, una cosa es decir drogas no y otra mira, si... es que si tomas drogas no podrás hacer esto, no podrás hacer lo otro o te costará más esto, te costará más aprender conocimientos. (Pt/NR/F)

Por lo tanto, lo que está de moda en este mini grupo es fumar. $Y$ entonces, es esta cosa de utilizarlo al revés... lo que sabemos es que está condicionando a que la gente fume. (Pm/NA/M)

\section{El entorno como modulador de las prácticas en salud}

Tal y como se ha tratado en los apartados anteriores, las y los profesionales de salud pública atribuyen la decisión última en la persona que ejecuta la acción. No obstante, entienden que el entorno es también un pilar esencial en el que se gesta la decisión. Sostienen que el entorno limita o posibilita el desarrollo de ciertas prácticas según las condiciones materiales (por ejemplo, la accesibilidad a productos, o las circunstancias del ambiente) pero, también, las condiciones sociales, políticas, económicas e ideológicas (ámbito donde especialmente se trabajan las desigualdades en salud).

Al considerar el entorno como un factor que modula, potenciando o interfiriendo la toma de decisiones personales en salud, las y los profesionales de salud pública sugieren en las entrevistas que las intervenciones en promoción de la salud no pueden basarse únicamente en la educación personal, por el contrario exigen integrar el entorno como objeto de intervención.

En este sentido, las y los profesionales de la salud, a la meta de capacitar a las personas en la toma de decisiones con relación a su salud agregan una segunda meta complementaria: trabajar sobre el entorno entendido como elemento modulador que permite (o no) la toma de decisiones y el ejercicio de las prácticas en salud.

Así es la promoción: es facilitar. Y fíjate que vemos en la definición de Ottawa, capacitar a los individuos de la comunidad porque ellos son los responsables de su salud. Pero nosotros facilitamos, lo tenemos que hacer fácil... pero los protagonistas son ellos [...] la sociedad tiene que facilitar la promoción de la salud global. (Pm/NA/M) 
Partiendo de una configuración central del sujeto como unidad mínima de incidencia en la salud poblacional, agregan dos grandes niveles de apelación del entorno, como capas de influencia que recubren y modelan a la persona: su entorno próximo (grupos de relación) y los determinantes que generan desigualdades en salud:

Yo creo que tenemos que dar un mensaje, una estrategia de intervención con la que pueda contemplarlo todo ino? Yo doy un consejo que interpele a la persona respecto a lo que hace, miraría algo relacionado con el ámbito más social o más comunitario y, después, otra miraría sobre el determinante, sobre las causas de las causas" (SP/NA/M)

Así pues, retornando a los argumentos de crear voluntades en salud, en las entrevistas se resalta la importancia de incidir sobre el entorno cercano de las personas como estrategia para facilitar y fomentar la realización de prácticas de salud, incrementar el acceso o penalizar informalmente las prácticas no saludables.

La gente sabe que no tiene que fumar [...] ya puedes saber pero si todos los lideres fuman pues, tu fumarás [...] Entonces estos elementos del entorno, este elemento de facilitación que te decía antes en los que tenemos que trabajar, que la gente quiera hacerlo. (Pm/NA/M)

Además, al entorno cercano le agregan una segunda capa que cubre al sujeto: los determinantes de la salud. Comprenden que tanto la comunidad como las condiciones sociales y económicas producen condiciones materiales que influyen en las posibilidades de diversos colectivos de realizar prácticas saludables, según su posición dentro del entramado social. Por ello, y en especial quienes tienen una mayor tradición en el trabajo sobre los determinantes sociales de la salud, destacan el rol del Estado en reducir las desigualdades sociales con el objetivo de equilibrar la balanza y reducir la brecha que impone una separación en el acceso a la salud por condiciones sociales, económicas y/o ideológicas para, en consecuencia, producir entornos que faciliten y fomenten la toma de decisiones saludables. Destacan pues, el trabajo sobre las desigualdades en salud.

Por lo tanto, el individuo está en el centro y tiene un papel de decisión que nunca se debe perder. Y lo que tenemos que ayudar es que cada vez tenga más capacidad de decisión, pero tenemos que hacer que todas las condiciones en las que vive, en las que trabaja... todos los determinantes estructurales de nivel socioeconómico [...] la situación de crisis, de paro, etc. Todo esto va en contra de la salud, por lo tanto, tenemos que ayudar a que la sociedad sea una sociedad que genere salud. (Pm/NA/M

\section{La convivencia de enfoques contrapuestos sobre la responsabilidad y sus efectos pragmáticos y éticos}

En la tensión entre los discursos sobre la persona como sujeto libre y autónomo que toma decisiones personales y el entorno como elemento que modula e incide, produciendo escenarios específicos que hacen que ciertas prácticas y voluntades de ser saludables sean posibles, las y los profesionales de salud pública constituyen tres enfoques sobre la relación entre la responsabilidad personal y la responsabilidad social-estatal. Estos tres enfoques no se unifican en un corpus único de ideas sobre la salud sino que, por el contrario, conviven juntos entre los diferentes profesionales que llevan a cabo acciones de salud pública.

En lo referente al primer enfoque, quienes se ubican en trayectorias de intervención relacionadas con la promoción de la salud y los determinantes sociales destacan que la situación socioeconómica contribuye en gran medida en determinar sus hábitos en salud. Bajo esta conceptualización, si bien 
no se despoja a la persona de su capacidad de acción (por el contrario, es sujeto de intervención como un valor a potenciar y a tener en cuenta en las políticas de salud pública) la toma de decisiones no saludables no es conceptualizada como una consecuencia inequívoca de actos personales, sino que tiene en cuenta los factores sociales y de sistema que determinan la toma de decisiones.

Plantean, pues, que no hay culpa personal en la acción, dado que cada sujeto actúa condicionado por la posición y situación social, política y económica que le predisponen para la acción.

\section{Hay muchos determinantes [...] toda una serie de cosas de manera que nunca, nunca, nunca culpabilizar a la persona. Culpabilizar a la víctima desde un punto de vista de sociedad, de determinantes sociales de la salud [...] su situación de salud puede estar determinada por el lugar en el que vive, por sus condi- ciones. $(\mathrm{Pm} / \mathrm{NA} / \mathrm{M})$}

En cuanto al segundo enfoque, las y los profesionales que ocupan posiciones técnicas dentro de la salud pública (con menor experiencia de trabajo en determinantes sociales de salud), destacan la importancia del establecimiento de responsabilidades mínimas e ineludibles del Estado. Tal concepción tiene una raíz práctica de distribución equitativa de salud, es decir, quienes defienden esta idea afirman que, ante los recursos limitados en salud, el Estado debe proporcionar servicios básicos a la totalidad de la población. Además, tiene otra raíz ideológica, puesto que la responsabilidad del Estado sería garantizar la supervivencia y velar por la salud de la población sobre ciertos problemas considerados fundamentales, mientras que contemplan otros como accesorios (parte de un lujo posible para quienes tengan recursos $\mathrm{e}$ interés en adquirirlos).

Es decir que te cubre a un nivel, tienes que esperar si te quieres operar de algo, pero tarde o temprano te lo harán. Te cubre el nivel básico, después si quieres ir a un médico mejor, con una técnica mejor, entonces te lo tienes que pagar. Pero al menos que las necesidades básicas estén cubiertas. (Pt/NR/F)

Finalmente, en el juego de articulación entre las responsabilidades personales y responsabilidades sociales-estatales, ciertos profesionales de salud pública centran sus discursos en el sujeto y en su autonomía para tomar decisiones, relegando los componentes sociales y estructurales a un plano secundario. En este sentido, las y los profesionales que se centran en las responsabilidades personales relativizan, hasta prácticamente invisibilizar, la responsabilidad social en la realización de ciertas prácticas y en el desarrollo de enfermedades.

Que entienda que su salud es una responsabilidad suya, no es una responsabilidad de la sociedad. Ahora seré muy bestia pero claro, si tú fumas durante cuarenta años estas asumiendo un riesgo. Lo que no vale es que cuando [...] se ha asumido este riesgo digas "hombre, es que además usted ahora me está maltratando, porque yo resulta que me tenía que operar de cáncer de pulmón y me lo está retrasando seis meses", " escuche, ¿perdón? usted lleva cuarenta años fumando ¿eh? ¡Yo no le he obligado a fumar a usted, no!" (Pt/NT/MA)

Este último enfoque, que desliga las elecciones personales de su contexto de producción, lleva a hiperresponsabilizar a la persona por su propio estado de saludenfermedad. Se trata de una hiperresponsabilización que se acerca a la culpa, puesto que confiere una carga moral a la decisión personal, como la causa de malestar o enfermedad.

Es un tema de responsabilidad personal. O sea, nos hemos autoacostumbrado y la administración durante estos veinte últimos años nos ha acostumbrado a pensar que todo lo que nosotros tenemos como problema es un problema que la 
administración se encarga [...]. Yo pienso que hay una responsabilidad ineludible que es tu responsabilidad personal, [...] la decisión final es tuya y por lo tanto la responsabilidad final es tuya. (Pt/NT/MA)

Con todo, hasta en los casos de hiperresponsabilización de las personas, las y los profesionales de salud pública destacan en las entrevistas otro elemento que actúa más allá del entorno y de las decisiones personales: la carga biológica. En este sentido, la culpa nunca llega a ser completa puesto que nuestro cuerpo, entendido como la estructura biológica que sustenta nuestro ser, actúa de forma diferenciada e independiente de nuestras intenciones.

Al final de todo es una cuestión de probabilidades. [...] es el precio que tenemos pagar por ser pluricelulares [...] pero si tienes un cierto rigor y una cierta disciplina, haces ejercicio y tienes cuidado con la alimentación, tal y tal, compras muchos menos billetes de lotería. (Pt/NT/MA)

\section{DISCUSIÓN}

En el trabajo evidenciamos dos pilares esenciales para el entendimiento de la promoción de la salud como una estrategia biopolítica en el contexto neoliberal| ${ }^{24,25)}$. En primer lugar, la producción de saberes legitimados en discursos de la evidencia científica como elemento clave para el gobierno de las subjetividades en materia de salud. Y, en segundo lugar, la construcción de las personas entendidas como la pieza mínima a partir de la cual la construcción de sociedades saludables ${ }^{(4,5)}$ es posible.

En relación con el primer pilar, Castiel et al. ${ }^{(26)}$ destacan la emergencia de una nueva moralidad sanitaria sustentada por los imperativos de la evidencia científica que, a partir de la noción del autocuidado, configura realidades y prescribe buenas prácticas y malas prácticas de las personas con su propia salud. Tal y como se ha expresado en la investigación, las y los profesionales de salud pública de Cataluña que formaron parte de la investigación sitúan al Estado como entidad que produce, maneja y gestiona el conocimiento experto sobre la salud poblacional. Por una parte, este aspecto le atribuye la responsabilidad de instrucción sobre la salud y sobre las estrategias para conseguirla a su población. Por otra parte, hace que sus discursos e intervenciones sobre la salud adquieran especial legitimidad(27), construyéndolos pues, como verdades inapelables que revelan a la población qué es y cómo ser saludable. En este sentido, la acción de informar lleva asociada la pretensión de convencer a quienes son informados/as, para así lograr cierta predisposición a actuar de forma acorde con el discurso oficial sobre la salud.

Con relación al segundo pilar, diversos autores críticos de la perspectiva individualista de los procesos de $\operatorname{salud}^{(28,29,30)}$ destacan que las estrategias de los gobiernos neoliberales anatomizan la comprensión de las prácticas sociales a partir del entendimiento de las personas como empresarias de sí mismas; sujetos racionales que eligen libremente su propio destino, a partir de sus competencias, conocimientos e intereses y orientan las acciones de gobierno a reforzar la idea la producción de sujetos disciplinados y expertos en la gestión de su salud ${ }^{(31)}$. Precisamente, la lógica que entiende al sujeto como un ser racional que toma sus decisiones a partir del conocimiento y la voluntad personal es el principal discurso que articula las acciones de promoción de la salud presentadas en el artículo. Así pues, las y los profesionales de salud de Cataluña enfatizan la importancia de producir y diseminar conocimientos sobre cuáles son las prácticas que hacen a la persona saludable y cómo alcanzarlas y, por otra parte, diseñan estrategias para guiar las intenciones personales hacia la voluntad de ser saludable.

Ahora bien, de acuerdo con las argumentaciones de Castiel ${ }^{(32)}$, Dumas et al. ${ }^{(33)} \mathrm{y}$ Gurrieri et al. ${ }^{(24)}$, es pertinente recalcar que el foco en la persona como sujeto responsable de su propia salud es excesivamente simplista por obviar la estructura social y las desigualdades 
sociales como determinantes de la salud, al mismo tiempo que lleva a la despolitización de las condiciones estructurales, materiales e históricas que permiten el desarrollo de las prácticas sociales (saludables o no). En nuestro caso, estos efectos se hacen especialmente evidentes en los posicionamientos expuestos por las y los profesionales que aluden a una responsabilidad mínima del Estado en garantizar ciertos servicios básicos de salud para la población, atribuyendo a la persona la responsabilidad de actuar para mejorar aquellas condiciones "accesorias". Esto resulta más evidente en aquellas afirmaciones que invisibilizan el papel de los condicionantes sociales y enfatizan la autonomía personal como eje esencial de la salud, lo cual deriva, tal y como se puede observar en el estudio presentado, en una cuasi culpabilización de la persona de su estado de salud o falta de ella.

Gurrieri et al. ${ }^{(24)}$ advierten que el discurso de la elección que promete el ejercicio de soberanía sobre uno mismo, a partir del ejercicio de la "libertad" y la "independencia" personal es, como mínimo, dudoso y desigual para aquellas personas que no disponen de recursos y condiciones que faciliten el ejercicio de prácticas hegemónicamente saludables. Aspectos que se traducen en divergencias sociales en la construcción de nociones sobre la salud personal y en la disponibilidad de medios para alcanzarla. Muestra de ello son los hallazgos de Dumas et al. ${ }^{(33)}$ que contraponen las nociones asociadas al cuidado en salud de personas de clase social acomodada a las nociones de personas en situación de vulnerabilidad económica; mientras las primeras conciben el cuidado del cuerpo y la salud como un fin en sí mismo, quienes se encuentran en condiciones desfavorecidas conciben el cuerpo y la salud como un medio para acceder a cubrir necesidades urgentes (alimentación, empleo, cuidado familiar, etc.). En este sentido, las nociones de cuidado en salud, como búsqueda de mejora y autorrealización personal $^{(34,35,36)}$ a partir de las cuales se legitiman las intervenciones de promoción de la salud centradas en la persona, obedecen a lógicas de entendimiento de la salud de una parcela de la población, justamente la más favorecida, y obvia las necesidades de buena parte de la población, dado que la urgencia de subsanar situaciones de precariedad y carencia destituye de relevancia del cuidado de la salud como fin en sí mismo.

Por su parte, Castiel ${ }^{(32)}$ argumenta que el foco en la persona y su consiguiente invisibilización y despolitización de los factores estructurales que hacen posible el ejercicio y acceso (o no) a la salud, colabora en el aumento de la estratificación social, puesto que divide a la población entre quienes pueden realizar el ejercicio de toma de decisiones entre múltiples y diversas opciones de ser saludables y quienes tienen un acceso reducido a ellas. No actuar sobre las condiciones estructurales naturaliza, pues, las situaciones de inequidad en salud a partir de la responsabilización de quienes se encuentran en situaciones de vulnerabilidad social de su propia condición.

Por ello, planteamos la imperiosa necesidad de integrar el entendimiento de las prácticas sociales como ejercicio de relaciones de poder siempre vinculado a la estructura social que la hace posible ${ }^{(37)}$, para resituar así los discursos de salud a partir de la imposibilidad de disociar las condiciones sociales, políticas y económicas con la producción de la salud personal y colectiva.

\section{CONCLUSIONES}

En el presente artículo nos hemos propuesto indagar las argumentaciones desplegadas por las y los profesionales de la salud de Cataluña en el ejercicio de pensar y diseñar intervenciones de promoción de la salud y sus efectos en la atribución de responsabilidades en salud. A partir de sus argumentaciones, hemos identificado a la persona y al Estado como los principales protagonistas en el desarrollo de estrategias para la construcción de sociedades saludables.

Hemos evidenciado que la configuración de la persona como eje a partir del cual se articulan las intervenciones de promoción de 
la salud deriva de su entendimiento como sujeto racional y libre que, a partir de diversos saberes, experiencias, criterios y situaciones, evalúa costos y beneficios y asume (o no) determinadas prácticas de cuidado de sí( ${ }^{(38)}$. Por su parte, encontramos que se constituye al Estado como poseedor de verdades en salud y responsable de instruir a la población sobre ellas. Es por ello que las y los profesionales de la salud participantes de la investigación remarcan el rol de las instituciones del gobierno en la provisión de saberes y criterios sobre la salud, que habilitan a las personas a valorar sus hábitos y su estado de salud, para actuar de forma más o menos coherente con ellos y así conseguir su propia mejora.

En este sentido, encontramos que las políticas e intervenciones de promoción de la salud proporcionan diversas tecnologías del yo que son, parafraseando a $\operatorname{Rose}^{(39)}$, formas en que las personas están capacitadas para actuar sobre sí mismas (sobre sus cuerpos, pensamientos, emociones y conductas) mediante el despliegue de saberes, criterios, estrategias, etc., en aras de mejorar su salud. Dicho sintéticamente, la acción del gobierno en promoción de la salud se dirige a generar saberes y condiciones que posibiliten que las personas asuman libremente el objetivo de alcanzar la salud y que, a su vez, estén dispuestas a aplicar ciertas tecnologías del yo para lograrlo.

En tercer lugar, encontramos que el entorno (material, político y social) es considerado como un elemento externo a la producción de salud, que influencia la asunción de las voluntades de realizar prácticas hegemónicamente saludables y las condiciones materiales que hacen posible tal realización. Aunque el foco en la persona, como agente último de toma de decisiones de su propia salud, es un elemento destacable en las argumentaciones expuestas por las y los profesionales de la salud, el peso que adquiere el entorno como condicionante de tales decisiones es divergente.

Observamos que el juego de las argumentaciones de contraposición entre la consideración del entorno como marco de posibilidades de ser saludables y de la persona como sujeto racional y autónomo tiene repercusiones en la atribución de responsabilidades en salud. Efectivamente, en las entrevistas se observa que, en los casos en que la elección personal es relativizada por la presión social y los condicionantes políticos, sociales, materiales y económicos, la responsabilidad en salud se vuelve una cuestión colectiva, en la que el Estado debe (además de informar e instruir a las personas sobre prácticas saludables) desplegar políticas correctivas de las desigualdades sociales y proporcionar herramientas para prevenir y solucionar los problemas de salud. Por el contrario, el acento en la capacidad de elección y la libertad individual como objeto último de intervención, construye la salud no como una experiencia simplemente vivida ${ }^{(8)}$, sino como un proceso sobre el que la persona puede incidir, mejorándola o empeorándola, a partir de las acciones y condiciones que ella misma haya escogido. Tal concepción puede derivar a una hiperresponsabilización del sujeto sobre sus prácticas y condiciones de salud: si la salud se elige, la falta de salud puede ser muestra de falta de responsabilidad, reflexividad o fuerza de voluntad de la persona ${ }^{(8)}$, dejando de esta manera, en un segundo plano, a los condicionantes sociales que limitan el marco de posibilidades a partir del cual tales decisiones pueden ser tomadas.

\section{AGRADECIMIENTOS}

Esta investigación pudo ser realizada gracias a la colaboración de la Agència de Salut Pública de Catalunya, Departament de Salut. Agradecemos especialmente a las y los profesionales que participaron en la investigación, y al Dr. Félix Vázquez Sixto, profesor titular del Departamento de Psico- logía Social de la Universidad Autónoma de Barcelona por sus reflexiones y su mirada crítica que orientaron el desarrollo de la tesis doctoral "La Salud Pública como problema de gobierno: Análisis de los problemas sociales de Salud Pública bajo el modelo de gobernanza" de la que deriva el presente artículo. 


\section{REFERENCIAS BIBLIOGRÁFICAS}

1. Palomo L, Ortún V, Benavides FG, Márquez-Calderón S. La salud pública frente a los efectos secundarios del progreso. Gaceta Sanitaria. 2006;20(Supl 1):209-221.

2. Catford J. Health promotion's record card: How principled are we 20 years on? Health Promotion International. 2004;19(1):1-4.

3. Llorca E, AmorMT, Merino B, Márquez FJ, Gómez F, Ramírez R. Ciudades saludables: una estrategia de referencia en las políticas locales de salud pública. Gaceta Sanitaria. 2010;24(6):435-436.

4. Kickbusch I. Responding to the health society. Health Promotion International. 2007;22(2):89-91.

5. Kickbusch I. Health governance: The health society. In: McQueen D, Kickbusch I, (ed.). Health and modernity: The role of theory in health promotion. New York: Springer; 2007.

6. Foucault M. Nacimiento de la biopolítica. Madrid: Ediciones AKAL: 2009.

7. Devisch I, Vanhuele S. Foucault at the bedside: a critical analysis of empowering a healthy lifestile. Journal of Evaluation in Clinical Practice. 2015;21:427-432.

8. Greco M. Thinking beyond polemics: approaching the health society through Foucault. Österreichische Zeitschrift für Soziologie. 2009;34(2): 13-27.

9. Marta CB, Teixeira ER. Genealogia do cuidado na perspectiva foucaultiana. Fundamental Care Online. 2016;8(3):4749-4756.

10. Rabinow $\mathrm{P}$, Rose N. Thoughts on the concept of biopower today [Internet]. 2003. [citado $28 \mathrm{feb}$ 2016]. Disponible en: http://tinyurl.com/h4a7znx.

11. Catalunya. Llei 18/2009, del 22 d'octubre, de salut pública. Diari Oficial de la Generalitat de Catalunya. 2009;(5495):81070-81116.

12. Santoro Lamelas V. La salud pública en el continuo salud-enfermedad: un análisis desde la mirada profesional. Revista de Salud Pública. 2016;18(4):530-542.

13. Vázquez F. La memoria como acción social: Relaciones, significados e imaginario. Barcelona: Paidós Ibérica; 2001.

14. Gergen KJ. Toward transformation in social knowledge. New York: SpringerVerlag; 1982.
15. Iñiguez L. Nuevos debates, nuevas ideas y nuevas prácticas en la psicología social de la era 'post-construccionista'. Athenea Digital. 2005;(8). doi: 10.5565/rev/athenead/v1n8.235.

16. Wetherell M, Potter J. Análisis del discurso y la identifiación de los repertorios interpretativos. En: Gordo López AJ, Linaza JL, (comp.). Psicologías, discursos y poder (PDP). Madrid: Visor; 1996.

17. Garay A. Poder y subjetividad: Un discurso vivo [Tesis de doctorado]. Bellaterra: Universidad Autónoma de Barcelona; 2001.

18. Ruiz Olabuénaga JI. Metodología de la investigación cualitativa. Bilbao: Publicaciones de la Universidad de Deusto; 2012.

19. Denzin NK, Lincoln YS. Handbook of qualitative research. Londres: Sage; 2000.

20. Holstein JA, Gubrium JF. The active interview. Londres: Sage; 1995.

21. Rubin HJ, Rubin IS. Qualitative interviewing: The art of hearing data. Los Angeles: Sage; 2005.

22. Braun V, Clarke V. Using thematic analysis in psychology. Qualitative Research in Psychology. 2006;3(2):77-101.

23. Coffey A, Atkinson P. Encontrar el sentido a los datos cualitativos: Estrategias complementarias de investigación. Medellín: Contus; 2003.

24. Gurrieri L, Brace-Govan J, Previte J. Neoliberalism and managed health: fallacies, façades and inadvertent effects. Journal of Macromarketing. 2014;34(4):532-538.

25. Kenny KE. The biopolitics of global health: Life and death in neoliberal time. Journal of Sociology. 2015;5(1):9-27.

26. Castiel DL, Santos Ferreira M, Ribeiro de Morales D. Os riscos e a promoção do autocontrole na saúde alimentar: moralismo, biopolítica e crítica parresiasta. Ciência \& Saúde Coletiva. 2014;19(5):1523-1532.

27. Ibáñez T. La Gobernanza: pieza clave del neoliberalismo avanzado. Libre Pensamiento [Internet]. 2012 [citado 16 abr 2016]. Disponible en: http://tinyurl.com/h7xz83q.

28. Mayes C. Governing through choice: Food labels and the confluence of food industry and public health discourse to create "healthy consumers". Social Theory \& Health. 2014;12:376-395. 
29. Crawshaw P. Governing at a distance: Social marketing and the (bio)politics of responsibility. Social Science \& Medicine. 2012;74(1):200-207.

30. McNay L. Self as enterprise: Dilemmas of control and resistance in Foucault's The Birth of Biopolitics. Theory, Culture and Society. 2009;26 (6):55-77.

31. Cronin JM, McCarthy M, Dalaney M. Deconstructing consumer discipline: How self-management is experience in the market place. European Journal of Marketing. 2015;49(11/12):1902-1922.

32. Castiel DL. O acesso aos Campos Elísios: a promoção da saúde ampliada e as tecnologias de melhoramento em busca da longevidade (e da inmortalidade). Saúde e Sociedade. 2015;24(3):1033-1046.

33. Dumas A, Robitaille J, Jette SL. Lifestile as a choice of necessity: young women, health and obesity. Social Theory \& Health. 2014;12(2): 138-158.
34. Rose N, O'Malley P, Valverde M. Gubernmamentality. Annual Review of Law and Social Science. 2006;6:83-104.

35. Rose N. Powers of freedom: Reframing political thought. Cambridge: Cambridge University Press; 1999.

36. Greco M, Stenner P. Happiness and the Art of Life: Diagnosing the psychopolitics of wellbeing. Health Culture and Society. 2013;5(1). doi: 10.5195/hcs.2013.147.

37. Cockerham WC. Bourdieu and an update of health lifestyle theory. In: Medical Sociology on the Move: New Directions in Theory. Dordrecht: Springer; 2013.

38. Foucault M. Tecnologías del yo y otros textos afines. Barcelona: Paidós Ibérica; 1990.

39. Rose N. Governing the soul: The shapong of the private self. New York: Routledge; 1990.

\section{FORMA DE CITAR}

Santoro Lamelas V. La persona y el Estado como agentes de responsabilidad para la producción de sociedades saludables: análisis temático desde la perspectiva de profesionales de la salud en Cataluña (España). Salud Colectiva. 2017;13(1):45-61. doi: 10.18294/sc.2017.1002.

Recibido: 5 de junio de 2016 | Versión final: 20 de octubre de 2016 | Aprobado: 20 de diciembre de 2016

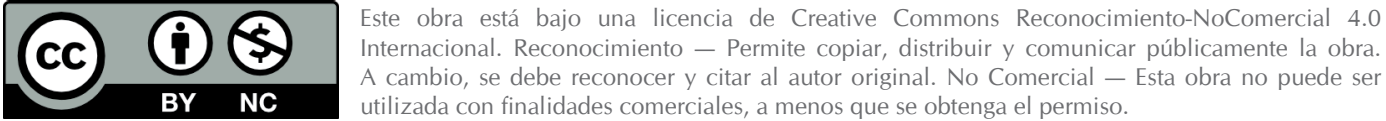

http://dx.doi.org/10.18294/sc.2017.1002 